\title{
A catalogue of soft $X$-ray sources in the galactic center region
}

\author{
L. Sidoli ${ }^{1}$, T. Belloni² ${ }^{2}$ and S. Mereghetti ${ }^{3}$ \\ 1 Astrophysics Division, Space Science Department of ESA, ESTEC, Postbus 299, 2200 AG Noordwijk, \\ The Netherlands \\ 2 Osservatorio Astronomico di Brera, Via Bianchi 46, 23807 Merate (Lc), Italy \\ 3 Istituto di Fisica Cosmica "G. Occhialini", CNR, via Bassini 15, 20133 Milano, Italy
}

Received 13 October 2000 / Accepted 15 December 2000

\begin{abstract}
We present a catalogue of 107 point-like $\mathrm{X}$-ray sources derived from a systematic analysis of all the ROSAT PSPC observations of the galactic center region performed in 1992-1993. Besides SgrA*, the massive black hole at the galactic center, $41 \mathrm{X}$-ray sources have been positionally associated with already classified objects. Twenty are identified with foreground stars and five with known Low Mass X-ray Binaries. The majority of the sources in our catalogue still remains unidentified. They are hard and/or severely absorbed and probably represent a large population of X-ray binaries located in the galactic center region, accreting at low accretion rates, and still largely unknown.
\end{abstract}

Key words. catalogues - galaxy: center - X-rays: general - X-rays: stars

\section{Introduction}

The galactic center (hereafter GC) region has always been among the privileged targets of many $\mathrm{X}$-ray missions. The observations made over the years established that a severe crowding of X-ray sources exists towards this part of our Galaxy (see Sidoli et al. 1999, for the results of a BeppoSAX survey of the GC with its Narrow Field Instruments). Many of the brightest sources are probably Low Mass X-ray Binaries (LMXBs) containing neutron stars or black holes, both with persistent and transient behavior, while the nature of the faintest sources is unknown. Many transient sources have been discovered in the last few years, especially during the monitoring of the galactic center region with the BeppoSAX Wide Field Camera (Ubertini et al. 1999) and the Rossi-XTE All Sky Monitor (Bradt et al. 2000).

The ROSAT Position Sensitive Proportional Counter (PSPC) performed a raster scan of the GC region $(|l|<$ $\left.1.5^{\circ}\right) \times\left(|b|<2^{\circ}\right)$ in 1992 and 1993 . The region covered is rectangular $\left(3^{\circ} \times 4^{\circ}\right)$ with the major axis oriented perpendicular to the galactic plane.

We performed a detailed spatial analysis of these data with the main objective of obtaining a catalogue of $\mathrm{X}$-ray sources in the soft X-ray energy band. All the data analyzed here have been retrieved from the ROSAT public archive in MPE. These datasets have previously been analyzed by Predehl \& Trümper (1994) and by

Send offprint requests to: L. Sidoli,

e-mail: lsidoli@astro.estec.esa.nl
Predehl et al. (1995), but neither of these works was aimed at the production of a full catalogue of sources in the region.

\section{Observations and data reduction}

The PSPC on board the ROSAT satellite (Pfeffermann et al. 1986) covered the energy band $0.1-2.4 \mathrm{keV}$ with a moderate energy resolution (3-4 energy bands can be defined) and an angular resolution of $\sim 20^{\prime \prime}$ (FWHM). The detector has a circular field of view of $\sim 2^{\circ}$ diameter. The radial and circular supports of the entrance window produce some artifacts, whose effects are mitigated by wobbling the satellite during pointing observations.

The data consist of 43 pointed observations with exposure times in the range 2000-3000 s, aimed at completely covering the central part of our Galaxy with the inner region of the PSPC detector. These observations were performed between 1992 February and 1993 March. We also included in our sample a single deeper pointing (47000 s) centered on the Sgr A* position performed on 1992 March 2. The log of the observations is reported in Table 1.

The data have been analysed using EXSAS (Extended Scientific Analysis System, version 98APR; Zimmermann et al. 1993) implemented in the ESO-MIDAS version 97NOVpl2.0 on Sun/Solaris.

All the analysis described below was performed in four different energy ranges: $0.1-2.4 \mathrm{keV}$ (channels 8-240, total energy band, $T$ ), $0.1-0.4 \mathrm{keV}$ (channels 8-40, soft energy 
Table 1. The PSPC observations log

\begin{tabular}{|c|c|c|c|}
\hline $\begin{array}{l}\text { Observation } \\
\text { ID }\end{array}$ & $\begin{array}{l}\text { Pointing Direction } \\
\text { RA \& Dec. (J2000) }\end{array}$ & $\begin{array}{l}\text { Obs. Start } \\
\text { dd/mm/yy hh:mm }\end{array}$ & $\begin{array}{l}\text { Obs. End } \\
\text { dd/mm/yy hh:mm }\end{array}$ \\
\hline rp400150n00 & $\begin{array}{llllll}17 & 49 & 16.80 & -30 & 12 & 00.0\end{array}$ & 04/03/92 03:48 & $04 / 03 / 92 \quad 04: 28$ \\
\hline rp400179n00 & $1747 \quad 57.60-300148.0$ & 02/03/92 19:53 & 02/03/92 20:41 \\
\hline rp400180n00 & $174638.40-295100.0$ & 02/03/92 08:49 & 02/03/92 09:25 \\
\hline rp400181n00 & $1745 \quad 19.20-294048.0$ & 02/03/92 01:11 & 02/03/92 03:03 \\
\hline rp400182n00 & $174400.00-293036.0$ & 01/03/92 20:06 & 01/03/92 20:45 \\
\hline rp400183a01 & $1742 \quad 43.20-291948.0$ & 28/03/93 19:56 & 28/03/93 20:38 \\
\hline rp400183n00 & $1742 \quad 43.20-291948.0$ & 02/03/92 11:54 & 02/03/92 12:25 \\
\hline rp400184n00 & $174124.00-290900.0$ & 01/03/92 10:26 & $10 / 03 / 92 \quad 12: 14$ \\
\hline rp400185n00 & 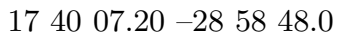 & 29/02/92 21:38 & $29 / 02 / 92 \quad 22: 26$ \\
\hline rp400186n00 & $173850.40-284800.0$ & 29/02/92 15:16 & 29/02/92 19:09 \\
\hline rp400187n00 & $1750 \quad 04.80-295436.0$ & 03/03/92 16:37 & 03/03/92 10:45 \\
\hline rp400188n00 & $1748 \quad 43.20-294424.0$ & 03/03/92 11:52 & 03/03/92 17:08 \\
\hline rp400189n00 & $\begin{array}{llllll}17 & 47 & 26.40 & -29 & 34 & 12.0\end{array}$ & 02/03/92 21:33 & $02 / 03 / 9222: 16$ \\
\hline rp400190n00 & 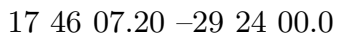 & 03/03/92 08:40 & 03/03/92 09:20 \\
\hline rp400191n00 & $174331.20-290300.0$ & 04/03/92 19:49 & 04/03/92 20:30 \\
\hline rp400192n00 & $\begin{array}{llllll}17 & 42 & 12.00 & -28 & 52 & 12.0\end{array}$ & 01/03/92 12:00 & 01/03/92 17:19 \\
\hline rp400193n00 & $174055.20-284200.0$ & 01/03/92 03:56 & 01/03/92 04:42 \\
\hline rp400194n00 & $\begin{array}{llllll}17 & 39 & 38.40 & -28 & 31 & 12.0\end{array}$ & 29/02/92 20:04 & 29/02/92 20:51 \\
\hline rp400195n00 & $1750 \quad 50.40-293748.0$ & 03/03/92 19:50 & 03/03/92 20:36 \\
\hline rp400196n00 & $174931.20-292700.0$ & 04/03/92 07:01 & 04/03/92 07:40 \\
\hline rp400197n00 & $\begin{array}{llllll}17 & 48 & 12.00 & -29 & 16 & 48.0\end{array}$ & 03/03/92 18:13 & 04/03/92 13:50 \\
\hline rp400198n00 & $174300.00-283524.0$ & 02/03/92 10:20 & 03/03/92 15:32 \\
\hline rp400199n00 & $174143.20-282436.0$ & 01/03/92 07:13 & 01/03/92 07:54 \\
\hline rp400200n00 & $174026.40-281424.0$ & 29/02/92 23:15 & 01/03/92 00:02 \\
\hline rp400201n00 & $175138.40-292024.0$ & 03/03/92 23:07 & 03/03/92 23:46 \\
\hline rp400202n00 & $\begin{array}{llllll}17 & 50 & 19.20 & -29 & 10 & 12.0\end{array}$ & 04/03/92 08:37 & 04/03/92 09:15 \\
\hline rp400203n00 & 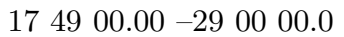 & 04/03/92 18:09 & 10/03/92 15:04 \\
\hline rp400204n00 & 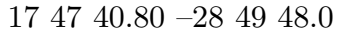 & 04/03/92 11:56 & 07/03/92 10:28 \\
\hline rp400205n00 & $174624.00-283900.0$ & 02/03/92 18:18 & 03/03/92 13:54 \\
\hline rp400206n00 & $1743 \quad 48.00-28 \quad 1800.0$ & 01/03/92 18:21 & 02/03/92 15:36 \\
\hline rp400207n00 & $1745 \quad 55.20-281124.0$ & 01/03/92 13:42 & 02/03/92 13:59 \\
\hline rp400208n00 & $1741 \quad 14.40-275700.0$ & 01/03/92 04:51 & 01/03/92 06:18 \\
\hline rp400209n00 & $175224.00-290300.0$ & 04/03/92 05:23 & 04/03/92 06:03 \\
\hline rp400210n00 & $1751 \quad 04.80-28 \quad 5248.0$ & 04/03/92 02:13 & $04 / 03 / 92 \quad 02: 53$ \\
\hline rp400211a01 & $174948.00-284236.0$ & 23/03/93 09:24 & 23/03/93 09:47 \\
\hline rp400211n00 & $\begin{array}{llllll}17 & 49 & 48.00 & -28 & 42 & 36.0\end{array}$ & 03/03/92 21:56 & 03/03/92 22:11 \\
\hline rp400212n00 & $\begin{array}{llllll}17 & 48 & 28.80 & -28 & 32 & 24.0\end{array}$ & 16/03/93 21:06 & 16/03/93 21:53 \\
\hline rp400213n00 & $\begin{array}{llllll}17 & 47 & 12.00 & -28 & 22 & 12.0\end{array}$ & 16/03/93 22:42 & $16 / 03 / 9323: 30$ \\
\hline rp400214n00 & $1745 \quad 55.20-281124.0$ & 02/03/92 07:06 & 02/03/92 07:49 \\
\hline rp400215n00 & $174436.00 \quad-2801 \quad 12.0$ & 01/03/92 21:34 & $01 / 03 / 9222: 21$ \\
\hline rp400216n00 & $1743 \quad 19.20-275100.0$ & 01/03/92 23:10 & 01/03/92 23:57 \\
\hline rp400217n00 & $1742 \quad 02.40-274012.0$ & 01/03/92 08:48 & 01/03/92 09:30 \\
\hline rp900162n00 & $174540.80-290000.0$ & 02/03/92 03:56 & 09/03/92 05:40 \\
\hline
\end{tabular}

band, $S$ ), 0.5-0.9 keV (channels 52-90, medium energy band, $M)$ and $0.9-2.4 \mathrm{keV}$ (91-240, hard energy band, $H)$.

\subsection{Source detection}

We first merged all the observations to produce mosaic images (with a binsize of $15^{\prime \prime}$ ) in the four energy bands, using only the inner part of the PSPC detector. In fact the pointing directions were appropriately defined to continuously map the region with the inner part of the detector, which provides the best sensitivity and angular resolution (adding the overlapping outer regions with a different
Point Spread Function would degrade the image quality). The corresponding exposure images were also produced in the four energy bands.

The final mosaic in the total energy band, corrected both for the different exposure times and for the vignetting, is displayed in Fig. 1. The corresponding (vignetted) exposure map, used to correct it, is shown in Fig. 2.

We then applied, for each energy band, a source detection algorithm based on the following steps:

- local-detection algorithm: consisting of a sliding window technique, where a detection cell of $3 \times 3$ pixels is 


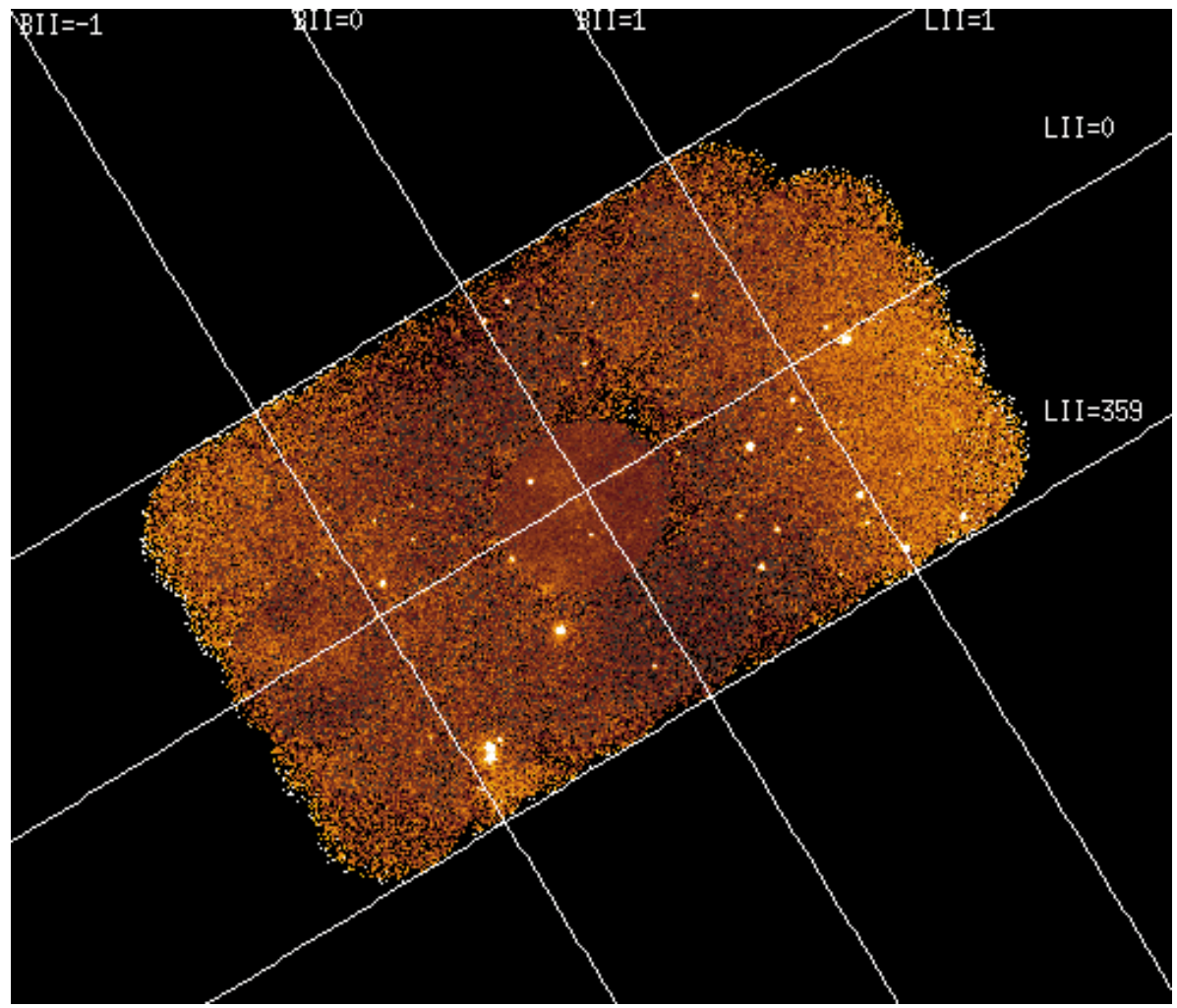

Fig. 1. Mosaic image of the galactic center region in the $0.1-2.4 \mathrm{keV}$ energy range. Only the inner part of the PSPC detector has been used. The image has been corrected for the exposure and for the vignetting

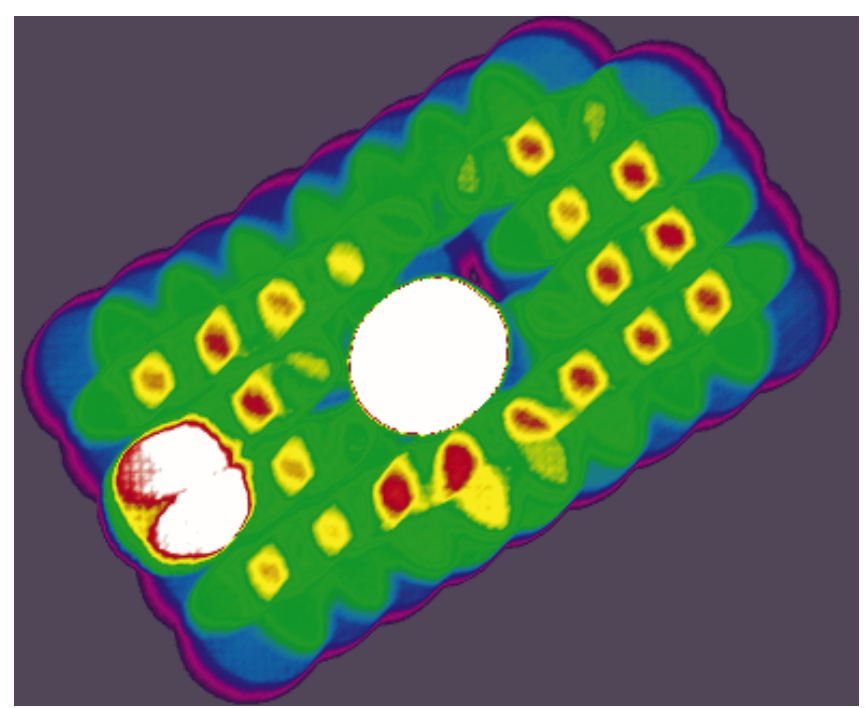

Fig. 2. Exposure map appropriate for the PSPC mosaic in the total energy band

shifted across the images. The total counts inside the detection cell are compared with a local background taken from the 16 pixels surrounding the detection cell itself. A $3 \sigma$ detection threshold has been applied. This local detection is used to produce a preliminary list of sources;

- production of a smoothed background image: the source list produced in the previous step is used to remove from the image circular regions around each source position. The resulting source-free image is fitted by a two-dimensional spline function to fill the holes and to produce a background image. A background image is produced for each energy band;

- map-detection algorithm: a new sliding window search is performed, this time using the background image to extract information about the local background. In other words, for each detection cell, the corresponding background is extracted, not from a frame surrounding it, but from the background map at that position. A second list of sources is produced, again with a $3 \sigma$ detection threshold;

- merging of the source lists: the two lists of sources resulting from the two sliding window searches are merged together, removing duplicates in order to have a unique list of sources (in each energy band). Duplicates are removed checking whether the distance between two sources is less than twice the sum of the size of the detection windows or less than the FWHM of the point spread function of the instrument;

- maximum-likelihood method: a maximum-likelihood method (Cruddace et al. 1987) is then applied to the photon lists, using the merged list of sources as 


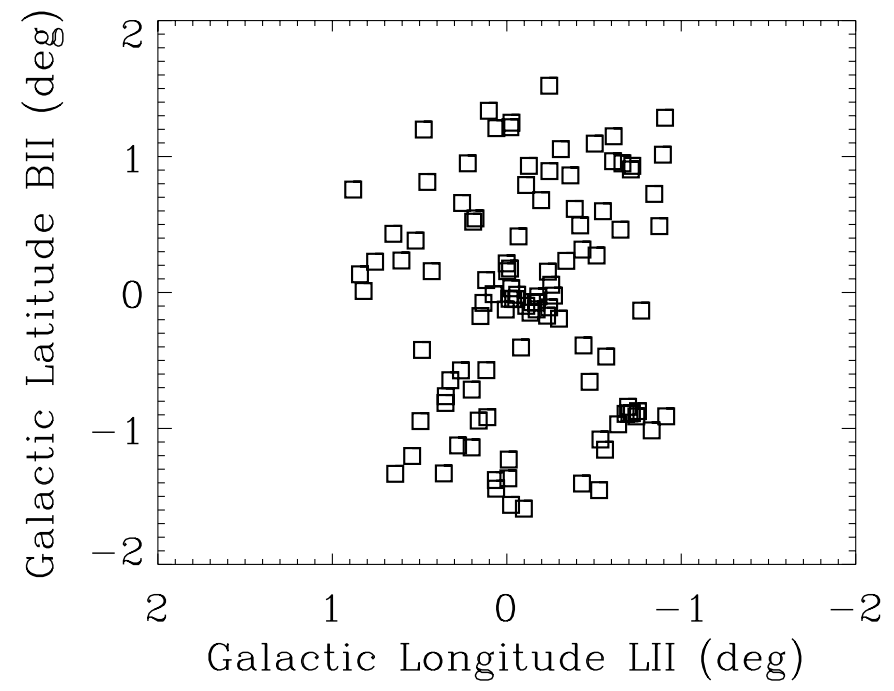

Fig. 3. Distribution of the ROSAT sources detected in the galactic center region

candidates. This method considers the ROSAT PSPC point spread function and the position of the source inside the detector and derives a source position and an existence likelihood. Only a detection likelihood larger than 10 (corresponding to a probability of a chance detection smaller than $\mathrm{e}^{-10}$ ) was considered as a true source. A final list of sources with their positions and positional uncertainties is thus produced.

This process yielded four lists of sources (one for each energy band).

The same procedure was also applied on the individual observations, this time also considering the external part of the PSPC detector, resulting in $4 \times 43$ lists of sources (4 energy bands and 43 observations). This second search was motivated by the fact that, e.g. due to source variability, some sources might have been missed in the previous global analysis.

Finally, all the lists of detected sources were cross correlated in order to clean the catalogue, removing all the sources with a multiple detection. By multiple detection we mean two or more sources whose position is compatible with each other, in which case only the source with higher existence likelihood has been kept. This procedure yielded a final catalogue containing 107 sources.

The count rates and upper limits $(2 \sigma)$ in each energy band have been extracted from the photon events tables at the position of the detected sources. Two softness ratios have been also derived: $S / H$ and $M / H$, where $H, M$ and $S$ are the net source counts in the hard, medium and soft energy bands defined in Sect. 2 .

\section{The catalogue of galactic center sources}

The spatial distribution of the sources detected in the surveyed region is displayed in Fig. 3. The remarkable symmetry of this distribution, and in particular the rather uniform source density as a function of galactic latitude, probably indicates that many sources are at a distance much closer than that of the galactic center. The final catalogue is reported in Table 3, where for each source the identification number, coordinates (J2000), count rate in the total energy band (0.1-2.4 keV), softness ratios $S / H$ and $M / H$ and possible identifications are reported. It is possible that a source has an upper limit in the total energy band, but is detected in one of the single energy bands. This is due to the energy-dependent background: faint sources detected, for example, in the hard energy range, could have been missed in the total energy band due to a higher background level. For these sources, the count rate in the energy band where a detection has been found is shown (and marked). On the other side, a few sources have been detected in the total energy band, but have only upper limits in all the other energy ranges; in this case the softness ratios are missing from Table 3 . In Figs. 4 and 5 the two softness ratios versus the count rate in the total energy band are shown.

A ROSAT PSPC count rate of 0.01 counts $\mathrm{s}^{-1}$ corresponds to about $510^{-11} \mathrm{ergs} \mathrm{cm}^{-2} \mathrm{~s}^{-1}$ (unabsorbed flux), assuming a $5 \mathrm{keV}$ bremsstrahlung spectrum and a column density of $610^{22} \mathrm{~cm}^{-2}$.

\section{Discussion}

The application of the maximum-likelihood method to 43 pointings of the $\mathrm{GC}$ region performed with the ROSAT PSPC instrument in 1992-1993 has led to a new catalogue of soft X-ray sources. Our catalogue contains 107 detections, down to a count rate of $\sim 0.001$ counts $\mathrm{s}^{-1}$ in the energy range $0.1-2.4 \mathrm{keV}$.

A correlation with the SIMBAD database resulted in probable identifications based on positional coincidence. The most plausible identifications (objects falling inside the PSPC error circle) are reported in Table 3. Other candidate counterparts (IRAS sources, for example) whose positions fall outside the PSPC error are also listed, when their large error boxes overlap with the ROSAT error box. For the possible counterparts listed in Col. (8) we give in parenthesis the distance between the optical and X-ray positions, the spectral type and magnitudes for stars, and other relevant informations as described in the notes to Table 3 .

\subsection{X-ray binaries}

Our discovery of ROSAT counterparts to a few previously known X-ray sources, allows us to significantly improve their positions. This is the case of the recently discovered 729 s X-ray pulsar AXJ 1740.2-2848 (Sakano \& Koyama 2000, later named AX J1740.1-2847 by Sakano et al. 2000) and of the other two ASCA sources AX J1744.3-2940 (Sakano et al. 1999a) and AX J1740.3-2904 (Sakano et al. 1999b).

We also inspected the error boxes of the X-ray binaries indicated in Table 2, without finding any ROSAT 


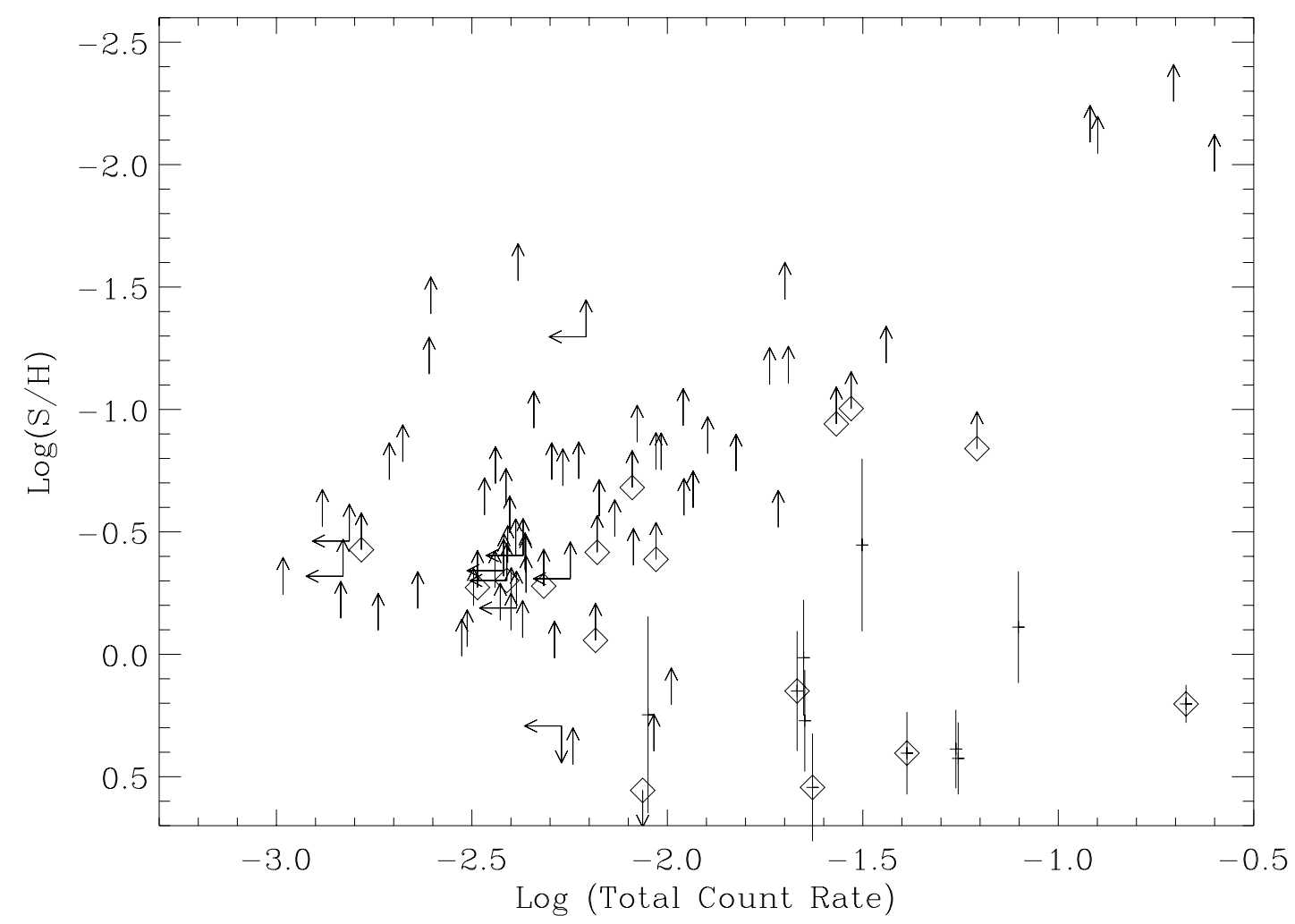

Fig. 4. Softness ratio $S / H$ as a function of the source count rate in the total energy band (0.1-2.4 keV). The upper limits on the total count rate refer to the sources that were detected only in the Soft or in the Hard energy bands. All the error bars are at $1 \sigma$ and the upper/lower limits at $2 \sigma$. The square symbols mark the sources possibly identified with stars

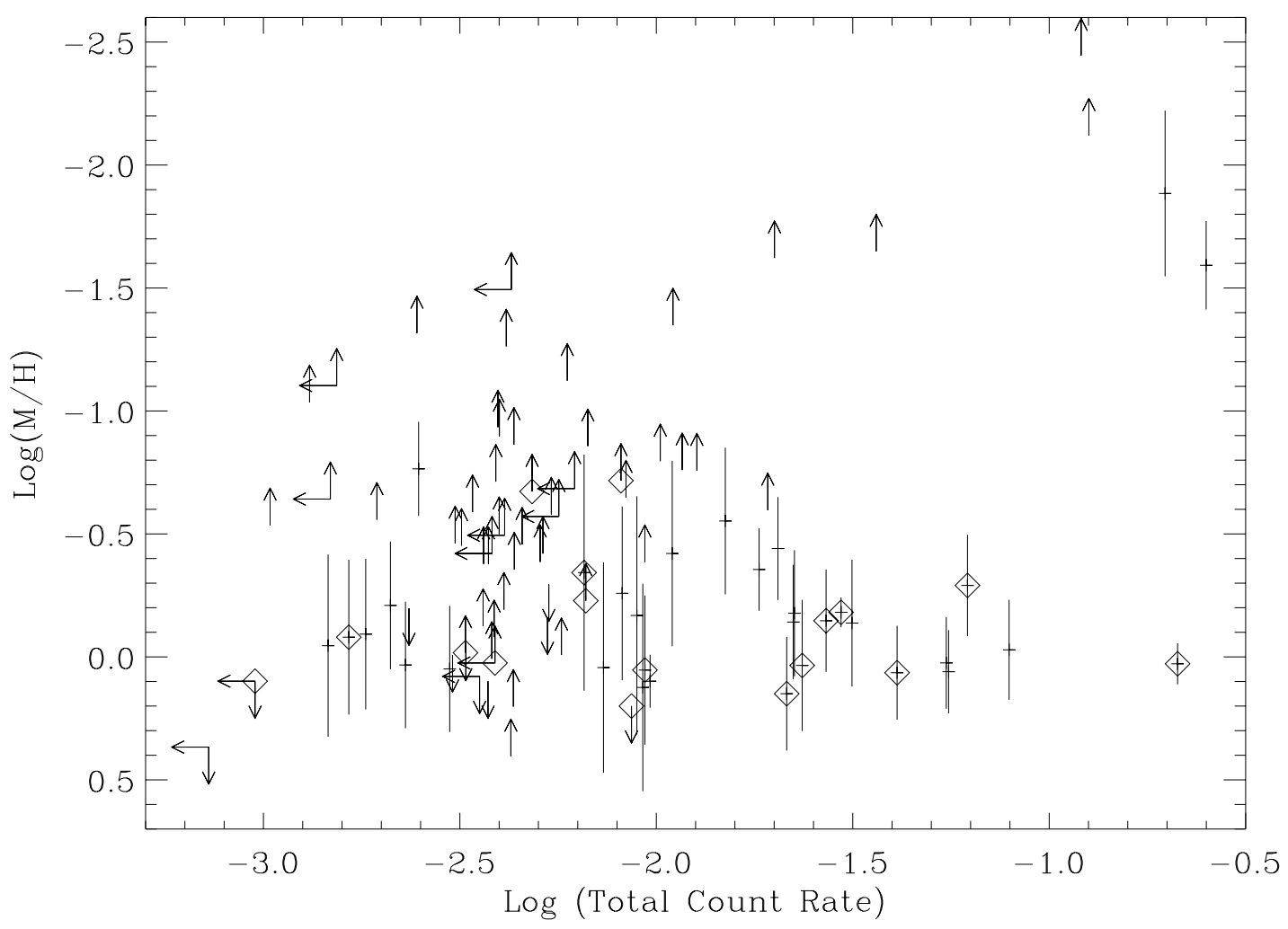

Fig. 5. Softness ratio $M / H$ as a function of the source count rate in the total energy band (0.1-2.4 keV). The upper limits on the total count rate refer to the sources that were detected only in the Medium or in the Hard energy bands. All the error bars are at $1 \sigma$ and the upper/lower limits at $2 \sigma$. The square symbols mark the sources possibly identified with stars 
Table 2. X-ray sources not detected

\begin{tabular}{|c|c|}
\hline Source & Reference \\
\hline 1E $1743.1-2843$ & Cremonesi et al. 1999 \\
\hline XTE J1748-288 & In 't Zand et al. 1998 \\
\hline KS 1741-293 & In 't Zand et al. 1990 \\
\hline GX+1.1-1.0 & Proctor et al. 1978 \\
\hline $\mathrm{GX}+0.2-1.2$ & Proctor et al. 1978 \\
\hline GRS 1741.9-2853 & Sunyaev et al. 1991 \\
\hline GRS 1734-29 & Sunyaev et al. $1991 b$ \\
\hline GRS $1743-290$ & Cordier et al. 1993 \\
\hline GRS 1747-312 & Pavlinski et al. 1994 \\
\hline GRS $1747-341$ & Cordier et al. 1993 \\
\hline 1E $1742.5-2845$ & Watson et al. 1981 \\
\hline 1E $1742.7-2902$ & Watson et al. 1981 \\
\hline 1E $1742.9-2849$ & Watson et al. 1981 \\
\hline 1E $1743.1-2852$ & Watson et al. 1981 \\
\hline 1E $1741.2-2859$ & Mitsuda et al. 1990 \\
\hline GC X-2 & Cruddace et al. 1978 \\
\hline $\mathrm{GC} \mathrm{X}-4$ & Cruddace et al. 1978 \\
\hline XTE J1755-324 & Remillard et al. 1997 \\
\hline GRO J1744-28 & Lewin et al. 1996 \\
\hline SAX J1750.8-2900 & Bazzano et al. 1997 \\
\hline 1E $1740.7-2942$ & Mirabel et al. 1992 \\
\hline SAX J1747.0-2853 = GX 0.2-0.2 & In 't Zand et al. $1998 \mathrm{~b}$ \\
\hline XTE J1739-285 & Markwardt et al. 1999 \\
\hline GRS 1739-278 & Paul et al. 1996 \\
\hline
\end{tabular}

counterparts in our catalogue, within a radius of $1^{\prime}$ of their position.

\subsection{Foreground stars}

A number of ROSAT sources have stellar counterparts in their error circles (see Table 3). To test these associations, we computed the $\log \left(f_{\mathrm{X}} / f_{\text {opt }}\right)$ as in Voges et al. (1999), using a constant conversion factor of $1.4810^{-11} \mathrm{ergs} \mathrm{cm}^{-2} \mathrm{cts}^{-1}$, appropriate for stellar sources (Fleming et al. 1995). The derived optical to X-ray flux ratios are displayed in Fig. 6 as a function of the count rate in the total energy band.

Stars usually have $\log \left(f_{\mathrm{X}} / f_{\text {opt }}\right)$ values in the range $[-6,-1]$, depending on the spectral type. Therefore, on the basis of the ratio between their $\mathrm{X}$-ray to optical flux, all these sources could indeed be stellar X-ray sources.

From Figs. 4 and 5 it can be seen that the sources tentatively identified with stars have relatively softer (or less absorbed) spectra.

\subsection{Supernova remnants}

We searched for the possible association of ROSAT sources with galactic supernova remnants.

The inspection of the Green catalogue of all the SNRs (Green 1998) located in the region shows that no ROSAT point sources in our catalogue fall inside the region covered by the following supernova remnants: G0.3+0.0, G0.9+0.1, G1.0-0.1 and G359.1-0.5.

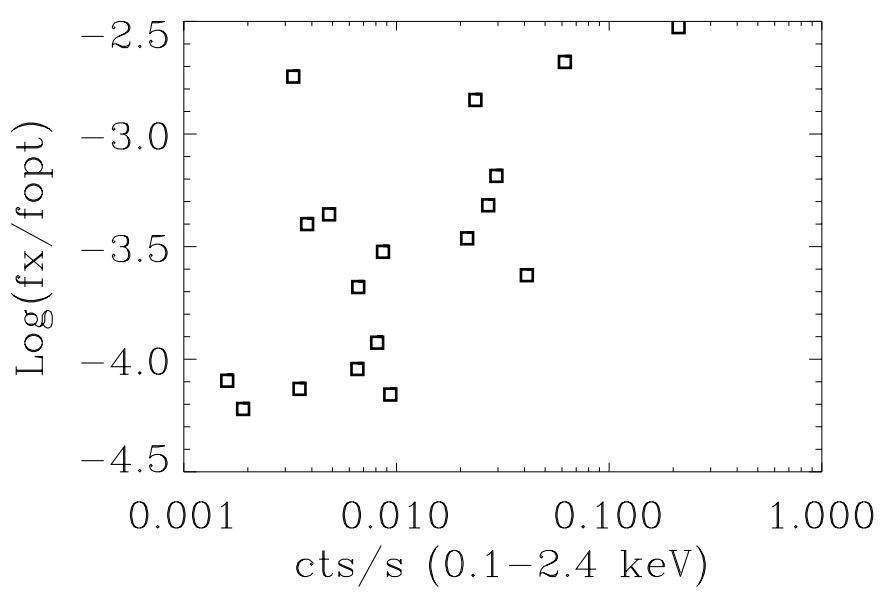

Fig. 6. $\log \left(f_{\mathrm{X}} / f_{\text {opt }}\right)$ as a function of the source count rate in the total energy band $(0.1-2.4 \mathrm{keV})$ for the sources identified with stars

Two ROSAT sources (n 81 and n 71) fall on or nearby the northern part of the radio shell of G359.0-0.9 $\left(23^{\prime}\right.$ size). Source $n 3$ is located at the center of the shell like SNR $359.1+0.9\left(11^{\prime} \times 12^{\prime}\right.$ size $)$. This source can also be associated with the star HD 316072 (see Sect. 4.2). Diffuse $\mathrm{X}$-ray emission has been recently detected with ASCA from the G359.0-0.9 shell and, marginally, from 359.1+0.9 (Sakano et al. 1999).

\section{Conclusions}

The spatial analysis, using a maximum-likelihood method, applied to public ROSAT PSPC data of the GC region led to the detection of 107 point-like sources, down to a PSPC count rate of 0.001 counts $\mathrm{s}^{-1}$ in the $0.1-2.4 \mathrm{keV}$ energy range.

Using the SIMBAD database, 42 sources have been associated with objects at other wavelengths; 20 are probably stars and 5 have been identified with previously known LMXBs. Other sources have been already classified as $\mathrm{X}$-ray objects, but their nature is still uncertain. Two sources in our catalogue could be associated with shells of supernova remnants and one with a molecular cloud or a maser source (n 72).

An error circle of $1^{\prime}$ radius centered on the positions of 25 LMXBs known to lie inside the region surveyed with PSPC has been inspected, leading to negative results.

All the sources brighter than 0.060 counts $\mathrm{s}^{-1}$ have been positionally associated with known objects.

The majority of our sources still remains unidentified. They are mostly undetected in the soft and medium PSPC energy ranges, they are quite hard and/or severely absorbed. Their average count rate in the total energy band $\left(0.01\right.$ counts $\left.^{-1}\right)$ translates into a luminosity in the range $10^{35}-10^{36} \mathrm{erg} \mathrm{s}^{-1}$ (assuming a distance of $8.5 \mathrm{kpc}$ ) which is suggestive of mass-transfer from a companion star onto a compact object in a binary system.

We propose that these still unidentified sources belong to a large population of X-ray binaries located in 
Table 3. The ROSAT PSPC Catalogue. The numbers in parenthesis in the first column refer to the sources detected by Predehl \& Trümper (1994). In Col. 5 sources not detected in the total $(T)$ energy band, but only in a partial $(S, M$ or $H)$ band, are labelled with a capital letter. Column 8 reports the probable identifications based on the cross-correlation with SIMBAD database; in parenthesis we indicate the offset, spectral type, $B$ and $V$ magnitude for stars. The meaning of the other symbols is: $\mathrm{Al}^{*}=$ Eclipsing Binary of Algol type; $\mathrm{V}^{*}=$ variable star; $\mathrm{bL}^{*}=$ Eclipsing Binary of beta Lyr type; ${ }^{*}{ }^{*}=$ star in double system; Em* $=$ emission line star; IR $=$ infrared source

\begin{tabular}{|c|c|c|c|c|c|c|c|}
\hline $\begin{array}{l}\text { Source } \\
\text { ID }\end{array}$ & $\begin{array}{l}\text { RA } \\
(\mathrm{J} 2000)\end{array}$ & $\begin{array}{l}\text { Dec } \\
(\mathrm{J} 2000)\end{array}$ & $\begin{array}{c}\text { Error } \\
\left({ }^{\prime \prime}\right)\end{array}$ & $\begin{array}{c}\text { cts ks }{ }^{-1} \\
(0.1-2.4 \mathrm{keV})\end{array}$ & $\log (S / H)$ & $\log (M / H)$ & Notes \\
\hline 1 & 173826.3 & -290147.2 & 6.2 & $79 \pm 6.93$ & $-0.111 \pm 0.228$ & $-0.0287 \pm 0.203$ & RX J1738.4-2901 \\
\hline 2 & 173908.6 & -282032.5 & 14.9 & $11.1 \pm 2.27$ & - & - & \\
\hline 3 & 173931.1 & -290950.3 & 8.9 & $61.9 \pm 5.86$ & $<-0.840$ & $-0.291 \pm 0.206$ & HD $316072\left(3^{\prime \prime} ; \mathrm{K} 0 ; B=11.2 ; V=9.97\right)$; at the center of G359.1+0.9 \\
\hline 4 & 173940.9 & -285111.3 & 10.0 & $6.61 \pm 1.36$ & $<-0.416$ & $<-0.229$ & V846 Oph $\left(1^{\prime \prime} ;\left(\mathrm{Al}^{*} \mathrm{~V}^{*}\right) \mathrm{A} 2 ; B=10.32 ; V=9.90\right)$ \\
\hline 5 & 174009.2 & -284723 & 11.4 & $5.06 \pm 1.17$ & $<-0.713$ & $<-0.386$ & AXJ $1740.2-2848$ (pulsar ) \\
\hline 6 & 174015.9 & -290332.1 & 10.0 & $19.2 \pm 2.54$ & $<-0.519$ & $<-0.597$ & AXJ 1740.3-2904 \\
\hline 7 & 174019.9 & -29004.90 & 9.8 & $4.34 \pm 1.30$ & $<-0.334$ & $<-0.863$ & \\
\hline 8 & 174023.2 & -290358.2 & 20.9 & $5.73 \pm 1.52$ & $<0.451$ & $<-0.00757$ & \\
\hline 9 & 174024.0 & -285650.5 & 3.6 & $55.4 \pm 3.23$ & $0.425 \pm 0.147$ & $0.0601 \pm 0.169$ & 1RX SJ174024.6-285700 $\left(18^{\prime \prime}\right)$ \\
\hline 10 & 174041.2 & -280850.0 & 16.0 & $3.73 \pm 1.2$ & - & $>0.0988$ & \\
\hline 11 & 174042.7 & -281813.4 & 1.4 & $251 \pm 6.75$ & $<-1.97$ & $-1.59 \pm 0.180$ & 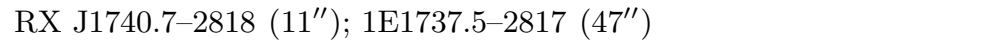 \\
\hline 12 & 174045.6 & -291631.3 & 11.9 & $10.2 \pm 2.15$ & $<0.2067$ & $<-0.796$ & \\
\hline 13 & 174046.7 & -283848.1 & 9.8 & $9.24 \pm 1.73$ & $<0.395$ & $0.124 \pm 0.422$ & \\
\hline 14 & 174051.4 & -281856.0 & 14.8 & $4.26 \pm 1.32$ & $<-0.0684$ & $<0.404$ & \\
\hline 15 & 174104.4 & -28154.3 & 5.0 & $22.3 \pm 2.14$ & $0.0140 \pm 0.236$ & $-0.141 \pm 0.232$ & \\
\hline 16 & 174123.7 & -284743.0 & 9.1 & $8.92 \pm 1.60$ & $0.247 \pm 0.402$ & $-0.169 \pm 0.484$ & \\
\hline 17 & 174133.8 & -284035.0 & 5.9 & $20.3 \pm 1.92$ & $<-1.107$ & $-0.441 \pm 0.209$ & \\
\hline 18 & 174136.7 & -292530.6 & 22.5 & $4.32 \pm 1.22$ & $<-0.345$ & $<0.202$ & \\
\hline 19 & 174141.8 & -283322.1 & 6.4 & $22.4 \pm 2.08$ & $0.271 \pm 0.208$ & $-0.177 \pm 0.256$ & \\
\hline 20 & 174158.0 & -290534.5 & 5.0 & $21.5 \pm 1.97$ & $0.15 \pm 0.24$ & $0.150 \pm 0.231$ & HD $160572\left(2^{\prime \prime} ; \mathrm{F} 3 \mathrm{~V} ; B=9.59 ; V=9.16\right)$ \\
\hline 21 & 174206.5 & -275412.5 & 18.7 & $3.07 \pm 0.9$ & - & - & \\
\hline 22 & 174215.2 & -291459.2 & 3.2 & $54.70 \pm 3.43$ & $0.387 \pm 0.16$ & $0.0245 \pm 0.187$ & \\
\hline 23 & 174216.9 & -28371.2 & 16.9 & $3.99 \pm 1.00$ & $<-0.203$ & $<-0.897$ & \\
\hline 24 & 174217.5 & -285647.6 & 11.6 & $3.98 \pm 1.11$ & $<-0.099$ & $<-0.499$ & \\
\hline 25 & 174227.8 & -281455.9 & 13.0 & $2.1 \pm 0.7^{(H)}$ & $<-0.342$ & $<-0.421$ & \\
\hline 26 & 174230.3 & -284456.3 & 1.8 & $212 \pm 6.65$ & $0.203 \pm 0.077$ & $0.0280 \pm 0.0836$ & V2384 Oph $\left(1^{\prime \prime} ;\left(\mathrm{bL}^{*} \mathrm{~V}^{*}\right) \mathrm{G} 3 / \mathrm{G} 5 \mathrm{~V} ; B=9.75 ; V=9.02\right)$ \\
\hline 27 & 174241.3 & -290213.6 & 8.7 & $11.6 \pm 1.45$ & $<-0.599$ & $<-0.760$ & \\
\hline 28 & 174319.5 & -29141.10 & 15.7 & $5.40 \pm 1.14$ & $<-0.688$ & $<-0.579$ & \\
\hline 29 & 174321.0 & -290829.9 & 19.2 & $3.1 \pm 0.99$ & $<-0.0307$ & $<-0.462$ & \\
\hline 30 & 174332.7 & -280725.0 & 5.5 & $23.5 \pm 1.9$ & $0.544 \pm 0.220$ & $0.0348 \pm 0.267$ & HD $316199\left(2^{\prime \prime} ; \mathrm{K} 5 ; B=10.6\right)$ \\
\hline 31 & 174340.2 & -282227.7 & 52.0 & $1.9 \pm 0.7^{(H)}$ & $<-0.302$ & $<0.0246$ & HD $316212\left(47^{\prime \prime} ; K 5 ; B=11.3 ; V=9.9\right)$ \\
\hline 32 & 174351.1 & -284643.7 & 11.6 & $11.0 \pm 1.94$ & $<-0.934$ & $-0.420 \pm 0.377$ & \\
\hline 33 & 174353.8 & -290621.9 & 15.1 & $2.7 \pm 1.0^{(H)}$ & $<-0.308$ & $<-0.571$ & IRAS $17407-2904\left(44^{\prime \prime}\right)$ \\
\hline 34 & 174355.6 & -282957.9 & 14.1 & $4.09 \pm 1.23$ & $<-0.402$ & $<-0.192$ & IR [OF84] $18\left(37^{\prime \prime}\right) ;$ IRAS 17407-2829 $\left(49^{\prime \prime}\right)$ \\
\hline 35 & 174403.1 & -28301.5 & 15.3 & $5.33 \pm 1.4$ & - & $>-0.295$ & \\
\hline 36 & 174417.6 & -293948.0 & 6.6 & $14.9 \pm 1.79$ & $<-0.748$ & $-0.553 \pm 0.298$ & AX J1744.3-2940 $\left(19^{\prime \prime}\right)$ \\
\hline
\end{tabular}


Table 3. continued

\begin{tabular}{|c|c|c|c|c|c|c|c|}
\hline $\begin{array}{l}\text { Source } \\
\text { ID }\end{array}$ & $\begin{array}{l}\text { RA } \\
(\mathrm{J} 2000)\end{array}$ & $\begin{array}{l}\text { Dec } \\
(\mathrm{J} 2000)\end{array}$ & $\begin{array}{c}\text { Error } \\
\left({ }^{\prime \prime}\right)\end{array}$ & $\begin{array}{c}\text { cts ks }{ }^{-1} \\
(0.1-2.4 \mathrm{keV})\end{array}$ & $\log (S / H)$ & $\log (M / H)$ & Notes \\
\hline 37 & 174427.4 & -290329.7 & 8.4 & $2.9 \pm 0.356$ & $<0.00717$ & $0.0489 \pm 0.256$ & \\
\hline 38 & 174446.2 & -274727.3 & 17.4 & $5.3 \pm 2.0$ & - & $>-0.161$ & \\
\hline 39 & 174447.0 & -284928.3 & 17.3 & $1.27 \pm 0.316$ & - & - & \\
\hline 40 & $1744 \quad 47.3$ & -290727.9 & 13.9 & $2.3 \pm 0.355$ & - & $>-0.198$ & \\
\hline 41 & 174453.3 & -285139.3 & 10.0 & $2.8 \pm 0.4^{(H)}$ & $<-0.404$ & $<-1.494$ & IR MGM 1-3 $\left(6^{\prime \prime}\right)$ \\
\hline $42(4)$ & 174500.1 & -285124.9 & 15.0 & $3.9 \pm 0.436$ & $<-0.495$ & $<-0.934$ & $1 \mathrm{E} 1741.7-2850\left(56^{\prime \prime}\right)$ \\
\hline 43 & 174503.8 & -291047.8 & 16.2 & $1.2 \pm 0.279$ & - & - & IR $\operatorname{GCS} 13\left(18^{\prime \prime}\right)$ \\
\hline $44(13)$ & 174518.1 & -290621.4 & 10.5 & $1.3 \pm 0.243$ & $<-0.522$ & $<-1.036$ & \\
\hline 45 & 174522.5 & -281732.5 & 15.1 & $3.62 \pm 1.24$ & $<-0.273$ & $<-0.126$ & \\
\hline 46 & 174526.1 & -285632.6 & 10.9 & $0.38 \pm 0.10^{(M)}$ & - & $>0.0986$ & CSI-28-17423 ( $\left.7^{\prime \prime} ; \mathrm{B} ; B=11.5 ; V=11.2\right) ; \mathrm{HD} 316223\left(7^{\prime \prime} ; \mathrm{G} ; B=1.3\right)$ \\
\hline 47 & 174528.0 & -29124.5 & 26.2 & $1.2 \pm 0.3^{(H)}$ & $<-0.462$ & $<-1.103$ & IRAS $17422-2911\left(51^{\prime \prime}\right)$ \\
\hline 48 & 174529.0 & -280925.1 & 10.3 & $8.37 \pm 1.60$ & $<-0.866$ & $<-0.648$ & \\
\hline $49(12)$ & 174530.3 & -290706.8 & 2.3 & $9.6 \pm 0.499$ & $<-0.753$ & $0.0989 \pm 0.108$ & \\
\hline $50(8)$ & 174532.5 & -285947.6 & 25.6 & $<0.30$ & - & - & \\
\hline 51 & 174532.6 & -284717.3 & 11.4 & $1.4 \pm 0.275$ & $<-0.147$ & $-0.0456 \pm 0.371$ & \\
\hline $52(11)$ & 174533.1 & -290550.0 & 8.0 & $1.0 \pm 0.220$ & $<-0.2446$ & $<-0.536$ & \\
\hline 53 & 174539.4 & -291733.6 & 16.4 & $0.9 \pm 0.2^{(H)}$ & $<-0.3189$ & $<-0.642$ & \\
\hline $54(7)$ & 174540.7 & -290029.4 & 13.7 & $2.46 \pm 0.48$ & $<-1.145$ & $<-1.316$ & $\operatorname{Sgr}^{*}\left(9^{\prime \prime}\right)$ \\
\hline 55 & 174541.7 & -290852.7 & 17.4 & $0.33 \pm 0.10^{(M)}$ & - & $>0.367$ & \\
\hline 56 & 174543.1 & -285936.7 & 18.7 & $5.5 \pm 0.5$ & $<-1.297$ & $<-0.684$ & \\
\hline $57(14)$ & 174544.0 & -291322.9 & 11.2 & $1.6 \pm 0.289$ & $<-0.427$ & $-0.0802 \pm 0.315$ & HD $316232\left(6^{\prime \prime} ; \mathrm{O}+\ldots ; B=11.1, V=10.4\right)$ \\
\hline $58(9)$ & 174544.1 & -290459.4 & 5.5 & $2.2 \pm 0.287$ & $<-0.1869$ & $0.0329 \pm 0.257$ & \\
\hline $59(6)$ & 174545.6 & -285829.2 & 4.8 & $2.4 \pm 0.286$ & $<-1.39$ & $-0.765 \pm 0.191$ & \\
\hline $60(3)$ & 174550.7 & -285243.7 & 5.9 & $2.1 \pm 0.275$ & $<-0.786$ & $-0.209 \pm 0.259$ & \\
\hline $61(10)$ & 174552.5 & -290749.4 & 6.6 & $1.8 \pm 0.266$ & $<-0.0979$ & $-0.0926 \pm 0.306$ & \\
\hline 62 & 174601.8 & -282916.7 & 16.4 & $8.64 \pm 1.5$ & $>0.556$ & $>0.199$ & HD $316297\left(11^{\prime \prime} ; \mathrm{K} 7 ; B=11.4 ; V=10.0\right)$ \\
\hline 63 & 174605.5 & -293054.8 & 3.2 & $121 \pm 4.37$ & $<-2.091$ & $<-2.446$ & A1742-294 $\left(13^{\prime \prime}\right)$ \\
\hline 64 & 174606.2 & -29409.40 & 13.0 & $3.27 \pm 0.80$ & $<-0.272$ & $<-0.0168$ & V734 Sgr $\left(4^{\prime \prime} ;\left(\mathrm{Al}^{*} \mathrm{~V}^{*} ?\right) ; B=13.00\right)$ \\
\hline $65(5)$ & $\begin{array}{lll}17 & 46 & 07.3\end{array}$ & -285950.2 & 5.7 & $1.9 \pm 0.271$ & $<-0.713$ & $<-0.558$ & \\
\hline 66 & 174608.2 & -281756.4 & 12.5 & $5.12 \pm 1.27$ & - & - & \\
\hline $67(2)$ & 174614.1 & -285144.9 & 18.2 & $4.1 \pm 0.623$ & $<-1.53$ & $<-1.26$ & 1E $1742.9-2849\left(30^{\prime \prime}\right)$ \\
\hline 68 & 174631.6 & -281029.0 & 13.5 & $3.90 \pm 1.15$ & $<-0.374$ & $<-0.713$ & \\
\hline $69(1)$ & 174639.2 & -285352.4 & 1.8 & $29.5 \pm 0.864$ & $<-1.00$ & $-0.181 \pm 0.060$ & HD $316314\left(0^{\prime \prime} ; \mathrm{F} 0 ; B=9.94 ; V=9.51\right) ; 1 \mathrm{E} 1743.4-2852\left(2^{\prime \prime}\right)$ \\
\hline 70 & 174700.4 & -29131.90 & 8.2 & $18.2 \pm 1.42$ & $<-1.102$ & $-0.355 \pm 0.167$ & \\
\hline 71 & 174700.8 & -301127.1 & 25.6 & $8.8 \pm 2.54$ & - & - & inside the shell of the SNR G359.0-0.9 \\
\hline 72 & 174703.7 & -29412.70 & 17.1 & $3.27 \pm 1.05$ & - & $>-0.0539$ & OH359.5-0.7(13"; Molecular Cloud); [TVH89]203(15"; Maser $)$ \\
\hline
\end{tabular}


Table 3. continued

\begin{tabular}{|c|c|c|c|c|c|c|c|}
\hline $\begin{array}{l}\text { Source } \\
\text { ID }\end{array}$ & $\begin{array}{l}\text { RA } \\
(J 2000)\end{array}$ & $\begin{array}{l}\text { Dec } \\
(J 2000)\end{array}$ & $\begin{array}{c}\text { Error } \\
(")\end{array}$ & $\begin{array}{c}\text { cts ks }^{-1} \\
(0.1-2.4 \mathrm{keV})\end{array}$ & $\log (S / H)$ & $\log (M / H)$ & Notes \\
\hline 73 & 174705.3 & -280854.8 & 6.4 & $27.0 \pm 2.81$ & $<-0.941$ & $-0.147 \pm 0.208$ & BN Sgr $\left(5^{\prime \prime} ;\left(\mathrm{Al}^{*} \mathrm{~V}^{*}\right) \mathrm{F} 3 \mathrm{~V} ; B=9.60 ; V=9.28\right)$ \\
\hline 74 & 174714.9 & -300157.5 & 17.7 & $3.6 \pm 1.2$ & $<-0.697$ & $<-0.378$ & \\
\hline 75 & 174715.5 & -295804.3 & 8.0 & $20 \pm 2.48$ & $<-1.450$ & $<-1.62$ & G359.23-0.92 (The Mouse) \\
\hline 76 & 174723.5 & -300039.3 & 22.4 & $36.2 \pm 3.61$ & $<-1.189$ & $<-1.649$ & \\
\hline 77 & 174725.4 & -300240.7 & 3.6 & $126 \pm 6.0$ & $<-2.046$ & $<-2.12$ & SLX $1744-300\left(27^{\prime \prime}\right)$ \\
\hline 78 & 174725.9 & -295957.8 & 2.7 & $197.2 \pm 7.30$ & $<-2.258$ & $-1.88 \pm 0.337$ & SLX $1744-299\left(15^{\prime \prime}\right)$ \\
\hline 79 & 174729.9 & -295857.7 & 17.3 & $11.0 \pm 2.09$ & $<-0.567$ & $<-1.348$ & \\
\hline 80 & 174731.0 & -281346.8 & 5.5 & $31.4 \pm 3.64$ & $-0.446 \pm 0.353$ & $-0.138 \pm 0.258$ & \\
\hline 81 & 174737.3 & -301023.9 & 27.6 & $12.6 \pm 3.12$ & $<-0.8195$ & $<-0.758$ & near the shell of the SNR G359.0-0.9 \\
\hline 82 & 174754.2 & -29598.4 & 15.3 & $4.1 \pm 1.25$ & - & - & \\
\hline 83 & 174807.8 & -290758.8 & 13.9 & $4.82 \pm 1.13$ & $<-0.279$ & $<-0.673$ & CD-29 $14004\left(7^{\prime \prime} ; \mathrm{B}+\ldots ; B=11.83 ; V=11.05\right)$ \\
\hline 84 & 174825.1 & -284424.5 & 15.7 & $3.19 \pm 0.84$ & $<-0.200$ & $<-0.452$ & \\
\hline 85 & 174828.7 & -290033.0 & 12.8 & $3.81 \pm 0.96$ & $<-0.319$ & $<0.00770$ & \\
\hline 86 & 174835.3 & -295723.3 & 10.7 & $8.11 \pm 1.36$ & $<-0.681$ & $<-0.717$ & HD $316341\left(6^{\prime \prime} ;\left(\mathrm{Em}^{*}\right) \mathrm{O}+\ldots ; B=9.54 ; V=9.06\right)$ \\
\hline 87 & 174849.4 & -300103.9 & 10.7 & $5.1 \pm 1.02$ & $<0.0156$ & $<-0.420$ & \\
\hline 88 & 174853.4 & -29086.70 & 7.5 & $8.19 \pm 1.27$ & $<-0.363$ & $-0.259 \pm 0.353$ & \\
\hline 89 & 174854.4 & -285939.2 & 11.0 & $6.55 \pm 1.31$ & $<-0.0571$ & $-0.343 \pm 0.480$ & HD $316308\left(9^{\prime \prime} ; \mathrm{K} 0 ; V=9.0\right)$ \\
\hline 90 & 174925.8 & -290157.2 & 16.7 & $1.9 \pm 0.7^{(H)}$ & $<-0.189$ & $<-0.494$ & \\
\hline 91 & 174928.6 & -291858.8 & 4.10 & $41.0 \pm 2.7$ & $0.404 \pm 0.169$ & $0.0645 \pm 0.191$ & $\begin{array}{l}\text { HD } 161907\left(6^{\prime \prime} ;\left(*^{*}{ }^{*}\right) ; B=8.33 ; V=8.05\right) \\
\text { CD-29 } 14038 \mathrm{~B}\left(9^{\prime \prime} ;\left({ }^{*}{ }^{*}\right) ; V=13.0\right)\end{array}$ \\
\hline 92 & 174937.8 & -290324.1 & 10.5 & $9.35 \pm 1.54$ & $<-0.754$ & $<-0.386$ & \\
\hline 93 & 174941.4 & -291712.7 & 17.3 & $3.5 \pm 1.02$ & - & - & HD $316418\left(16^{\prime \prime} ; \mathrm{F} 0 ; B=9.94 ; V=9.46\right)$ \\
\hline 94 & 175004.9 & -300829.3 & 16.4 & $7.33 \pm 1.61$ & $<-0.480$ & $0.0434 \pm 0.428$ & \\
\hline 95 & 175007.3 & -300155.2 & 14.1 & $3.73 \pm 1.17$ & $<-0.139$ & $<-0.378$ & \\
\hline 96 & 175024.9 & -293453.5 & 16.4 & $1.5 \pm 0.50^{(M)}$ & - & $>0.0794$ & \\
\hline 97 & 175029.4 & -29007.2 & 10.3 & $5.93 \pm 1.22$ & $<-0.717$ & $<-1.123$ & 1RXP J175029.8-285957 (10") \\
\hline 98 & 175033.8 & -292111.4 & 15.3 & $2.6 \pm 0.8$ & - & - & \\
\hline 99 & 175041.3 & -291644.5 & 7.5 & $9.35 \pm 1.38$ & $<-0.388$ & $0.0536 \pm 0.304$ & HD $162120\left(3^{\prime \prime} ;\left(*^{*} *\right)\right.$ A2V; $\left.B=8.51 ; V=8.33\right)$ \\
\hline 100 & 175058.0 & -293900.1 & 13.3 & $3.24 \pm 1.05$ & - & - & \\
\hline 101 & 175111.1 & -293541.9 & 9.8 & $3.41 \pm 0.99$ & $<-0.570$ & $<-0.589$ & \\
\hline 102 & 175125.9 & -293744.4 & 11.0 & $4.56 \pm 1.17$ & $<-0.924$ & $<-0.457$ & \\
\hline 103 & 175136.7 & -290531.8 & 17.1 & $3.02 \pm 0.86$ & - & $>0.0793$ & \\
\hline 104 & 175138.5 & -295026.3 & 14.8 & $6.69 \pm 1.93$ & $<-0.563$ & $<-0.857$ & \\
\hline 105 & 175141.4 & -291849.5 & 12.6 & $4.35 \pm 1.28$ & $<-0.252$ & $<-0.356$ & \\
\hline 106 & 175142.2 & -294547.4 & 31.0 & $3.5 \pm 1.1^{(S)}$ & $>0.293$ & - & \\
\hline 107 & 175221.2 & -290432.2 & 10.9 & $3.9 \pm 1.12$ & $<-0.608$ & $<-0.0804$ & \\
\hline
\end{tabular}


the GC region, accreting at low accretion rates, and still largely unknown. The transient sources recently discovered with ASM-XTE and WFC-BeppoSAX would be only the high luminosity tail of this population of LMXBs.

Acknowledgements. We have made use of the ROSAT Data Archive of the Max Planck Institut für extraterrestrische Physik at Garching; and of the SIMBAD database operated at Centre de Données astronomiques in Strasbourg. We thank A. Parmar for reading this manuscript and providing helpful comments. L. Sidoli acknowledges an ESA Fellowship.

\section{References}

Bazzano, A., Heise, J., Ubertini, P., et al. 1997, IAU Circ., 6597 Bradt, H., Levine, A. M., Remillard, R. A., \& Smith, D. A. 2000, in X-ray Astronomy 1999, Stellar Endpoints, AGN and the Diffuse Background, Bologna, Sep. 1999, ed. G. Malaguti, G. Palumbo, \& N. White (pub. Gordon and Breach) in press [astr-ph/0003438]

Cordier, B. 1993, in The Second Compton Symposium AIP 304, ed. C. E. Fichtel, N. Gehrels, \& J. P. Norris, New York, 446

Cremonesi, D. I., Mereghetti, S., Sidoli, L., \& Israel, G. L. 1999, A\&A, 345, 826

Cruddace, R. G., Hasinger, G. R., \& Schmitt, J. H. M. M. 1987, in Astronomy from Large Databases, ed. F. Murtaugh, \& A. Heck, 177

Cruddace, R. G., Fritz, G., \& Shulman, S. 1978, ApJ, 222, L95

Green, D. A. 1998, In A catalogue of galactic Supernova Remnants, http://WWW.MRAO.CAM.AC.UK/SURVEYS/SNRS/

Fleming, T. A., Molendi, S., Maccacaro, T., \& Wolter, A. 1995, ApJS, 99, 701

In 't Zand, J. J., Heise, J., Smith, M., et al. 1998, IAU Circ., 6840

In 't Zand, J. J., Bazzano, A., Cocchi, M., et al. 1998b, IAU Circ., 6846

In 't Zand 1990, Adv. Space Res., 11, 187

Lewin, W. H. G., Rutledge, R. E., Kommers, J. M., et al. 1996, ApJ, 462, L39

Markwardt, C. B., Marshall, F. E., Swank, J. H., et al. 1999, IAU Circ., 7300
Mirabel, F., Rodriguez, L. F., Cordier, B., et al. 1992, Nature, 358,215

Mitsuda, K., Takeshima, T., Kii, T., \& Kawai, N. 1990, ApJ, 353,480

Paul, J., Bouchet, L., Churazov, E., \& Sunyaev, R. 1996, IAU Circ., 6348

Pavlinskii, M., Grebenev, S. A., \& Sunyaev, R. A. 1994, ApJ, 425, 110

Pfeffermann, E., Briel, U. G., Hippmann, H., et al. 1986, SPIE, 733,519

Predehl, P., \& Trümper, J. 1994, A\&A, 290, L29

Predehl, P. 1995, in Nuclei of Normal Galaxies, ed. R. Genzel, \& A. I. Harris, NATO ASI Ser. C, 445 (Dordrecht: Kluwer), 21

Proctor, R. J., Skinner, G. K., \& Willmore, A. P. 1978, MNRAS, 185, 745

Remillard, R., Levine, A., Swank, J., \& Strohmayer, T. 1997, IAU Circ., 6710

Sakano, M., Imanishi, K., Tsujimoto, M., et al. 1999a, ApJ, 520,316

Sakano, M., Yokogawa, J., Mukarami, H., et al. 1999b, in Proc. of the Japanese German Workshop on High Energy Astrophysics, ed. W. Becker, \& W. Itoh, MPE Report, 270, 113

Sakano, M., \& Koyama, K. 2000, IAU Circ., 7364

Sakano, M., Torii, K., Koyama, K., et al. 2000, PASJ, 52, in press [astro-ph/0008331]

Sidoli, L., Mereghetti, S., Israel, G. L., et al. 1999, ApJ, 525, 215

Sunyaev, R. A., Pavlinskii, M., Churazov, E., et al. 1991, SovA. Lett., 17, 42

Sunyaev, R. A., Churazov, E., Gilfanov, M., et al. 1991b, Adv. Space Res., 11, 177

Te Lintel Hekkert, P., Versteege-Hansel, H. A., Habing, H. J., et al. 1989, A\&AS, 78, 399 (TVH89)

Ubertini, P., Bazzano, A., Cocchi, M., et al. 1999, Proc. Third INTEGRAL Workshop, Taormina 1998, Astroph. Lett. Comm., 38, 301

Voges, W., Aschenbach, B., \& Boller, Th. 1999, A\&A, 349, 389

Watson, M. G., Willingale, R., Grindlay, J. E., et al. 1981, ApJ, 250,142

Zimmerman, H. U., Becker, W., Belloni, T., et al. 1994, EXSAS User's Guide, MPE Report 257 